\title{
Lumen
}

Selected Proceedings from the Canadian Society for Eighteenth-Century Studies

\section{Rousseau et la conscience démocratique}

\section{Philip Knee}

Volume 23, 2004

URI : https://id.erudit.org/iderudit/1012196ar

DOI : https://doi.org/10.7202/1012196ar

Aller au sommaire du numéro

Éditeur(s)

Canadian Society for Eighteenth-Century Studies / Société canadienne d'étude du dix-huitième siècle

ISSN

1209-3696 (imprimé)

1927-8284 (numérique)

Découvrir la revue

Citer cet article

Knee, P. (2004). Rousseau et la conscience démocratique. Lumen, 23, 221-233. https://doi.org/10.7202/1012196ar

Copyright @ Canadian Society for Eighteenth-Century Studies / Société canadienne d'étude du dix-huitième siècle, 2004
Ce document est protégé par la loi sur le droit d'auteur. L'utilisation des services d'Érudit (y compris la reproduction) est assujettie à sa politique d'utilisation que vous pouvez consulter en ligne.

https://apropos.erudit.org/fr/usagers/politique-dutilisation/ 


\section{Rousseau et la conscience démocratique}

Je veux montrer que l'œuvre de Rousseau continue d'offrir des ressources pour réfléchir sur notre vie collective en démocratie. J'entends par cela moins les institutions de la démocratie, et le Contrat social comme l'une de leurs sources, que l'expérience de la démocratie, comme type de conscience, dont toute l'œuvre de Rousseau me semble annoncer et anticiper les enjeux. Je placerai mes remarques sous le patronage, si j'ose dire, de deux grands lecteurs de Rousseau qui ont été particulièrement attentifs aux enjeux psychologiques de l'expérience démocratique. Écrivant au début du XIX ${ }^{\mathrm{e}}$ siècle, ils permettent d'identifier - en schématisant - deux manières de se rapporter à la naissance de celle-ci, qui soulignent, je crois, la force et l'actualité de la pensée rousseauiste. Il s'agit de Chateaubriand et de Tocqueville.

Quoique à peu près contemporains, ils n'ont pas dialogué. La seule référence que l'un fait à l'autre, à ma connaissance, se trouve sous la plume de Chateaubriand dans ses Mémoires d'outre-tombe. Elle est brève, mais elle dit bien les choses: "Alexis de Tocqueville a parcouru l'Amérique civilisée dont $j^{\prime}$ ai visité les forêts ${ }^{1} »$. Leurs deux trajectoires américaines, en effet, ne se croisent pas. L'un étudie le fonctionnement de la nouvelle société démocratique, l'autre s'attache à ce que celle-ci remplace et détruit. Pour l'un, l'Amérique est un laboratoire, permettant de réfléchir sur la démocratie et son caractère central, l'égalisation des conditions ; pour l'autre, la modernité (Chateaubriand est l'un des premiers à employer le terme en français ${ }^{2}$ ) est synonyme de vulgarité et il lui oppose la noble sauvagerie de la nature, la tradition de la religion chrétienne et, bien sûr, les indigènes. Chateaubriand observe ces derniers et il déplore, en se référant à la vie sauvage décrite par Rousseau, qu'ils soient déjà dénaturés ${ }^{3} ; c^{\prime}$ est pourquoi il préfère les imaginer, de manière

1 Chateaubriand, Mémoires d'outre-tombe, Paris, Librairie générale française, t. 1, 1973, p. 652.

2 Chateaubriand, Mémoires d'outre-tombe, t. 3, p. 416.

3 Chateaubriand, Mémoires d'outre-tombe, t. 1, p. 283. 
qu'ils puissent devenir les symboles de sa protestation contre la modernité. Tocqueville, lui, ne voit pas en Amérique de tels motifs de protester. Certes, la nouvelle société $l^{\prime}$ " «attriste» et le "glace», et il est «tenté de regretter la société qui n'est plus ${ }^{4} »$; mais il regarde franchement l'absence d'aristocratie, de grands artistes, de monuments en Amérique, et il tend ce miroir à la France pour qu'elle y contemple son avenir. Bref, ces deux aristocrates bousculés par l'histoire voient l'inévitabilité du changement, mais ils l'acceptent différemment : Chateaubriand à contrecœur, en se tournant vers ces forêts en lesquelles la civilisation moderne va pleurer ce qu'elle perd ; Tocqueville, avec regret peut-être, mais en consentant à ce que cette société exige de lui - au premier titre, de la comprendre et de l'accueillir, fût-ce avec une "crainte salutaire" ${ }^{5}$.

Si ces deux attitudes opposées nous semblent familières aujourd'hui encore, c'est sans doute parce qu'elles sont complémentaires : la protestation romantique contre une société vide accompagne le souffle d'égalité et de liberté qui anime cette société. Or, l'une des raisons de l'intérêt que l'on continue de porter à la pensée de Rousseau est qu'elle a combiné ces deux attitudes ; elle a montré que l'exigence démocratique et la plainte romantique participent de la même modernité. On a beaucoup commenté la contradiction entre ces deux composantes de la pensée de Rousseau : l'état de nature et le contrat social, le promeneur solitaire et l'éducation du citoyen. Mais on peut aussi s'attacher à leur combinaison, et c'est peut-être là qu'on trouve surtout l'héritage de Rousseau.

Dans cet esprit, je vais procéder en trois temps. J'évoquerai d'abord deux sentiments, la pitié et la sincérité, sur lesquels Rousseau fait reposer sa critique de la société moderne, et qui nourrissent une autocritique de cette société que nous connaissons bien. Puis j'évoquerai le double langage qu'il juge nécessaire de tenir pour guérir les hommes modernes ; un double langage que nous pratiquons, je crois, mais sans en avoir toujours une claire conscience. Je dirai, enfin, quelques mots de l'incertitude dont Rousseau s'efforce de protéger ses lecteurs, en les mettant en garde par conséquent contre un aspect central de l'expérience démocratique.

\section{L'autocritique}

Commençons par le diagnostic de Rousseau. Dans une société où les besoins et les désirs se multiplient, les dettes et les obligations poussent

4 Tocqueville, De la démocratie en Amérique II, Paris, Robert Laffont, 1985, p. 658.

5 Tocqueville, De la démocratie en Amérique II, p. 656. 
les hommes à cacher toujours mieux leur sentiment pour obtenir les satisfactions qu'ils recherchent. Ils se rapprochent les uns des autres par leurs dépendances mutuelles, mais se séparent par ce qu'ils se cachent les uns aux autres. Plus les rapports sont nombreux sur le plan des intérêts, plus ils sont faux sur le plan des sentiments. Et cet abîme entre l'égoïsme dans les rapports utilitaires et l'apparence dans la vie morale semble devoir s'accentuer toujours davantage. Contrairement à la plupart des penseurs de son siècle, Rousseau réagit en refusant tout aménagement de cette contradiction, et il appuie sa critique sur le seul socle qui lui paraît avoir la solidité nécessaire : celui des sentiments partagés.

Mais loin d'en être le promoteur naif, comme on le peint parfois, Rousseau a une conscience aiguë de ce qui mine ce partage. Peut-on sentir avec un autre homme et partager ses passions sans les objectiver et sans en faire un spectacle ? Dans ce cas, en effet, le partage se transforme en une distance avec le sentiment éprouvé. Ce sentiment risque de n'être plus qu'une expérience de soi-même dont l'autre n'est que le moyen ou le prétexte, et l'on n'est plus l'acteur d'un partage vécu mais le spectateur d'un partage représenté. De quoi jouit-on, par exemple, quand on contemple le spectacle d'un navire qui coule, à partir d'un lieu où l'on est à $l^{\prime}$ abri de tout danger, selon l'interrogation classique de Lucrèce ${ }^{6}$ ? Si l'on jouit de n'être pas sur le navire, d'être un spectateur du drame au lieu d'en être la victime, il s'agit d'un plaisir d'amour-propre, dans la mesure où il procède du fait de vivre hors de soi par un regard porté sur les autres et, dès lors, par leur regard imaginé sur soi. Rousseau voit s'incarner dans l'expérience théâtrale un tel plaisir à distance, cautionné et généralisé dans toute la société. Car en dépit des apparences, cette expérience isole le spectateur, qui au milieu du public se sent délié de toute responsabilité. Il est même content de lui-même par la fausse solidarité qu'il manifeste pour les victimes d'un drame fictif. En fait, ce spectateur ne reconnaît d'existence aux autres que par le spectacle que ceux-ci lui offrent ; et il se dupe en croyant sympathiser avec la souffrance observée. Loin d'y participer et d'être poussé par elle à l'action, il se réfugie dans la bonne conscience d'avoir partagé la souffrance de son prochain ${ }^{7}$.

6 Lucrèce, De la nature des choses, Paris, Garnier-Flammarion, liv. 2, 1964, p. 53.

7 On sait que, pour combattre cette hypocrisie, Rousseau oppose les fêtes au théâtre, il veut rendre les spectateurs acteurs, et faire que "chacun se voie et s'aime dans les autres afin que tous en soient mieux unis." Lettre à d'Alembert, CEuvres complètes, t. 5, p. 115. Toutes les références à Rousseau renvoient à l'édition en cinq tomes des $C E u v r e s$ complètes, Paris, Gallimard, 1959-1995. 
Aussi généreuse qu'elle se prétende, la morale rationnelle des philosophes des Lumières entérine cette distance théâtrale où s'anéantissent les liens naturels du sentiment. «On peut impunément égorger son semblable sous la fenêtre [du philosophe], écrit Rousseau ; il n'a qu'à mettre ses mains sur ses oreilles et s'argumenter un peu, pour empêcher la Nature qui se révolte en lui, de l'identifier avec celui qu'on assassine ${ }^{8} .{ }^{\prime}$ Cette morale fait des autres des êtres toujours relatifs à mon intérêt. Pour que l'autre se manifeste à moi comme réalité absolue, il faut redonner sa place à un mouvement spontané d'identification à la souffrance de l'autre, un mouvement «obscur et vif», écrit Rousseau, antérieur à toute réflexion, et qui a été étouffé par l'amour-propre. C'est la pitié. Elle se traduit par l'exigence d'une identité sentie entre les hommes, ou d'un "moi commun», selon la formule du Contrat social ${ }^{9}$, qui conteste le réalisme ou le cynisme des penseurs modernes de l'intérêt.

Rousseau aperçoit les difficultés - probablement insurmontables d'une telle politique de la pitié. Par exemple, en étant obnubilé par telle ou telle souffrance particulière, le citoyen risque de ne pas s'ouvrir au bien commun. Il faut donc éduquer son sentiment dans le sens de la justice sociale, et cette généralisation de la pitié relève de la raison. Mais en étendant la pitié au-delà des cas particuliers, on peut vider la morale de son élan sentimental et en faire un amour froid de l'humanité en général. Cette sorte de dilution, que nous connaissons bien sous les traits de l'humanitarisme à notre époque, retrouve le danger initial que combattait Rousseau : celui d'une morale raisonnée qui transforme son objet en spectacle. Le sentiment d'humanité manifeste une généralisation de la pitié, et en un sens sa nécessaire rationalisation; mais du coup, en lui enlevant sa spontanéité, on l'affaiblit et on risque de l'éteindre.

La pitié tend à dépolitiser le lien social. Elle assure sans doute que l'autre homme ne soit pas subordonné à l'intérêt particulier, mais pas que cet homme soit présent comme homme, car avec la pitié il n'est qu'un être souffrant. La pitié évite l'écueil de l'amour-propre intéressé, mais elle ne reconnait pas la parole de l'autre, l'autre comme un être moral et politique. En faisant de la pitié le ressort du bien politique, on s'adresse au peuple comme à des malheureux, non comme à de futurs citoyens. Bref, à vouloir moraliser la politique par le sentiment, on risque de sentimentaliser la politique, au point de gommer sa spécificité comme lieu de délibération entre les différences. Mais n'est-ce pas précisément ce

8 Discours sur l'inégalité, CEuvres complètes, t. 3, p. 156.

9 Du contrat social, CEuvres complètes, t. 3, p. 361. 
caractère non politique de la pitié qui lui donne sa force d'attraction ? Elle permet à l'homme moderne de diriger un regard critique sur ses chefs et sur ses propres pratiques politiques, au point parfois de jeter l'opprobre sur la politique en général. Ce sentiment a donc une signification ambivalente : il est à l'origine de diverses dérives antipolitiques dans les sociétés modernes, mais aussi d'une précieuse capacité d'autocritique dont ces sociétés sont porteuses.

Une exigence connexe que Rousseau oppose aux sociétés modernes est la transparence. Elle se manifeste par l'idéal de sincérité qui anime ses œuvres autobiographiques et elle procède d'un certain échec de son projet politique initial. Il ne s'agit plus pour Rousseau de contrer la raison des Lumières en proposant un modèle social alternatif au leur, mais de s'adresser directement au lecteur pour toucher son cœur. En passant des "principes du droit» du Contrat social au récit de soi des Confessions, la stratégie rousseauiste reste axée sur l'exigence d'accorder les cœurs, mais elle se rétrécit et cherche à susciter le lien sentimental à partir du retrait et de la solitude.

La critique de l'hypocrisie n'a évidemment rien de spécifiquement rousseauiste. Ce qui l'est, c'est la manière dont Rousseau exalte pour elle-même la valeur de sincérité, au point que cette valeur en vienne à constituer en soi, pour bien des lecteurs, un projet moral et politique. À nouveau, ce lecteur est porté à faire de cette expérience un spectacle, en restant à distance d'elle pour la goûter passivement. Et Rousseau risque de jouer lui-même au jeu des fausses confidences, en livrant, si j'ose dire, une transparence spectaculaire. Dans les Confessions il apparaît comme à distance de lui-même, malgré ses affirmations répétées sur son unité personnelle, et son effort de transparence semble devoir échouer par l'exigence de sincérité qui l'anime.

Comme pour la pitié, tout se passe comme si Rousseau n'avait pas promu une valeur, mais surtout une aspiration qui s'avère vaine. En effet, n'est-ce pas l'échec de la sincérité qui, encore une fois, lui donne une si grande place dans la conscience de l'homme moderne ? Cet homme ne valorise-t-il pas la sincérité précisément parce qu'elle est absente de la société marchande ? Il n'oppose à sa propre existence, faite de masques et de calculs intéressés, cette valeur de sincérité ou d'authenticité, que parce qu'il trouve peut-être ainsi ce qu'il cherche vraiment : une posture d'autocritique permanente de ce qu'il est.

En somme, si les idées de souveraineté populaire ou de volonté générale ont nourri la pensée politique moderne, en même temps l'esprit de soupçon et l'exigence de pureté de Rousseau ont alimenté une sorte de moralisme anti-politique. Au nom de la pitié et de la transparence, s'est constituée une mauvaise conscience ou une contreculture, qui sont devenues inhérentes à la vie des sociétés démocra- 
tiques $^{10}$. Il en découle à la fois, et peut-être indissociablement, une certaine complaisance sentimentale et une vigilance morale face à l'hypocrisie et à la violence.

\section{Le double langage}

On peut identifier un second aspect de l'héritage de Rousseau, lui aussi, grâce aux attitudes contrastées de Chateaubriand et de Tocqueville face à la démocratie. Dans le Génie du christianisme, Chateaubriand dénonce la contamination de la France par les idées «libérales» du XVIIIe siècle. Mais sa pensée est, en fait, plus précise : ce ne sont pas ces idées comme telles qui sont nocives, mais la manière dont les penseurs des Lumières les ont mises de l'avant et diffusées, sans en comprendre les dangers. Selon Chateaubriand, les penseurs chrétiens du XVIIe siècle, comme Pascal, avaient déjà vu toutes les choses qu'allaient développer leurs successeurs, mais contrairement à ces derniers ils ont su les laisser voilées pour qu'elles n'entraînent pas de conséquences destructrices sur les esprits. Critiquant la religion et l'autorité, les philosophes des Lumières n'ont pas eu cette prudence, et ils ont contribué ainsi à miner la vie spirituelle et le sentiment d'appartenance ${ }^{11}$. Ils n'ont pas compris que, pour que la liberté ne se détruise pas elle-même, il faut s'adresser aux hommes par un double langage.

À l'inverse, Tocqueville observe que les sociétés démocratiques semblent fonctionner en prenant en charge le dévoilement dénoncé par Chateaubriand. À la fois admiratif et inquiet, Tocqueville s'interroge, par exemple, sur la religion utilitaire $e^{12}$ et sur le patriotisme réfléch $i^{13}$ par lesquels l'homme démocratique, du moins en Amérique, intègre l'ébranlement des certitudes du passé. L'âge démocratique procède de la mise en cause des références affectives partagées qui cimentent la société et qui lui permettent de se donner une âme commune ; mais loin de tout dissoudre et de tout balayer, la démocratie réussit à reconstituer un type de croyance qui assure un lien social renouvelé. Il n'est donc pas nécessaire d'utiliser un double langage pour protéger les citoyens contre eux-

10 Voir Arthur Melzer, "Rousseau and the Modern Cult of Sincerity», dans Clifford Orwin et Nathan Tarcov (édit.), The Legacy of Rousseau, Chicago, The University of Chicago Press, 1997, p. 291-292.

11 Chateaubriand, Génie du christianisme, Paris, Garnier-Flammarion, t. 1, 1966, p. $428-430$, et t. 2, p. 24-29.

12 Tocqueville, De la démocratie en Amérique I, p. 273-284.

13 Tocqueville, De la démocratie en Amérique I, p. 229-231. 
mêmes. Il faut reconnaître que chacun d'entre eux effectue déjà un tel dédoublement ; que la conscience démocratique est, en quelque sorte, une conscience dédoublée.

Au-delà du débat entre le traditionalisme et le libéralisme, c'est donc la diffusion des idées d'égalité et de liberté, et la capacité des hommes à les assumer ouvertement, qui est mise en jeu par Chateaubriand et Tocqueville. Or, ici encore, il me semble que Rousseau est allé au cœur du problème. Car il ne s'est pas contenté d'opposer son sentimentalisme à la pensée des Lumières ; il a minutieusement décrit les moyens politiques de sa mise en œuvre, en montrant que cette mise œuvre exige un double langage. En effet, si la sincérité est valorisée dans son œuvre autobiographique, elle est loin de l'être dans l'ensemble de sa pensée. Pourquoi?

L'élan vers l'autre homme, qui me lie à lui au-delà de tout raisonnement, exige d'être, non une idée, mais un élan justement, dont la force repose sur l'ignorance de soi. Les croyances ne cimentent la société que si elles ne sont pas pensées comme croyances. Une des conditions de la politique du sentiment est qu'elle ne soit pas comprise comme telle par ceux qui la portent. C'est pourquoi elle exige, de la part de ceux qui la mettent en œuvre, une stratégie de voilement. La politique rationnelle des philosophes des Lumières est inefficace, selon Rousseau, non seulement parce qu'elle les coupe de leurs sentiments naturels, mais aussi parce qu'elle ne prend pas la mesure du façonnement clandestin de la conscience sociale qui est nécessaire. Fabriquer le partage des sentiments, c'est le fabriquer pour les hommes et, d'une certaine façon, malgréeux.

Or, à travers l'image que les hommes ont d'eux-mêmes par les autres, on peut agir sur eux par le dedans, sur le plan de leurs désirs et de leurs espoirs, sans recourir au raisonnement ou à la contrainte. Les images que véhiculent les mythes et les fables ont le pouvoir de diriger les passions et de guider les pratiques. C'est pourquoi les vrais législateurs sont les poètes et les prophètes, qui savent faire vibrer les âmes et qui disposent les cœurs à aimer le bien commun. Pour que les hommes y concourent, il faut créer artificiellement une volonté publique, de sorte que chaque homme voie son propre bien dans le cadre de ce bien commun. Symboles patriotiques et religieux, persuasion, institutions répondant aux particularités historiques de chaque peuple, tout cela doit être mis en branle pour faire émerger entre les intérêts des points de jonction qui n'existaient pas naturellement, de sorte que soit amenée à l'existence ce que Rousseau appelle une volonté générale.

Dans le Contrat social, par exemple, la liberté consiste à vivre sous l'autorité des lois, en s'assurant que le peuple puisse ratifier ces lois et s'y reconnaître. Mais pour cela il doit être formé à ne plus les voir comme une dépendance sur le pouvoir d'autres hommes, et ceci passe par une 
ruse : que le peuple voie les lois comme ayant une origine divine ${ }^{14}$. Y consentir sur cette base illusoire rend les hommes libres. Rousseau pourrait dire qu'y consentir ainsi est rationnel, car c'est un moyen efficace de réaliser la fin recherchée : s'obéir à soi-même. Mais ce n'est pas un acte rationnel dans le sens d'un acte délibéré, guidé par la raison ; au contraire, les hommes consentent à ce qu'ils croient être des commandements divins, alors que les lois sont d'origine humaine. Cette dépendance dissimulée ou impersonnelle ne produit pas d'effets nocifs, car l'homme y retrouve le goût de son indépendance naturelle. Mais en vérité cette expérience n'est pas celle de l'indépendance, puisqu'elle dépend de ceux qui la façonnent.

On voit que l'enjeu est autant culturel que politique, car plus qu'un État, c'est un univers imaginaire qu'il s'agit de construire ; plus que des règles de fonctionnement universelles convenant à l'humanité en général, ce sont les conditions psychologiques du bonheur adaptées à chaque peuple qu'il faut mettre en place. Alors que les philosophes des Lumières prétendent tout éclairer au nom de la libération des superstitions, Rousseau fait d'une certaine illusion la condition de la liberté.

Certains de ses textes décrivent la liberté politique en la situant dans le cadre de l'illusion subjective qui lui est nécessaire ; d'autres décrivent les moyens de la fabrication de cette illusion. Je ne reprends pas ici le détail de ces descriptions. Je veux seulement souligner une question que soulèvent ces textes, qui est d'autant plus souvent négligée par les commentateurs que Rousseau lui-même ne la thématise pas : le citoyen peut-il vivre ensemble ces deux aspects de la politique ? Lui est-il possible de faire simultanément l'expérience de l'ordre politique comme un ordre moralement légitime, car garantissant la liberté des citoyens, et l'expérience de l'ordre politique comme un ordre fabriqué par des législateurs pour les citoyens à l'insu de ces derniers ${ }^{15}$ ?

Rousseau ne répond pas nettement, car il ne se demande jamais explicitement si un consentement politique acquis par la tromperie met en cause la liberté. Il procède plutôt comme si la liberté n'était accessible que par ce moyen. Les deux perspectives sont ainsi entremêlées. Or, on peut reconnaître dans cette ambivalence un aspect de l'expérience politique en démocratie, en considérant que c'est l'ambivalence de tous les citoyens. Tout se passe, en effet, comme si les citoyens démocratiques

14 Du contrat social, CEuvres complètes, t. 3, p. 383-384.

15 Pour une brève analyse du problème de la duplicité chez Rousseau, voir Philip Knee, «Images de Jean-Jacques : duplicité et liberté», Lumen, 20, 2001, p. 137-146. 
aspiraient à vivre en même temps les deux faces de la politique de Rousseau, en tenant à la fois un discours de liberté, manifestant un ordre de croyances qui les enveloppe, et un discours qui démystifie ces croyances et qui révèle leur fabrication.

Dans cette perspective, on peut dire que Rousseau anticipe les inquiétudes de Chateaubriand en explorant l'art politique de forger les croyances d'une société ; mais qu'il annonce aussi les descriptions plus sereines de Tocqueville, pour peu qu'on admette que le double langage des gouvernants ou des sages puisse devenir celui des gouvernés ou de l'ensemble des citoyens. En donnant à ce langage une place irréductible, Rousseau montre qu'il ne croit pas en un débat public régi par la seule raison ; mais il n'est pas pour autant machiavélien, si l'on entend par là que la liberté n'est qu'un vain mot hors des enjeux du pouvoir. Le double langage est inéliminable dans la mesure où l'ordre politique est un ordre de dépendances et d'apparences, auquel les hommes ne sauraient échapper ; mais c'est un ordre dont ils ne sont pas pour autant les dupes à tout moment.

La pensée de Rousseau aide donc à identifier deux aspects de la conscience démocratique : l'autocritique qui peut favoriser la vigilance morale, et le dédoublement qui peut favoriser la lucidité face aux excès du moralisme. Soit ; mais on conviendra que cette conscience ne tranche pas sur le contenu de la morale ou sur ce que le réalisme politique est en droit d'exiger. Elle garde cela dans une certaine indétermination, que le débat public, notamment, doit prendre en charge. C'est le troisième point que je veux aborder : l'incertitude ou le scepticisme de la conscience. Or, à cet égard, on ne peut plus se contenter de se nourrir librement des réflexions de Rousseau pour lui dessiner une postérité démocratique, comme je l'ai fait jusqu'ici. Il est nécessaire de mettre au premier plan l'intention de sa pensée, c'est-à-dire son souci d'anéantir les incertitudes de la conscience.

\section{L'incertitude}

Il y a bien un moment sceptique chez Rousseau, quand il fait la critique de la raison et des prétentions de la philosophie ou de la théologie à expliquer l'univers ${ }^{16}$. Il invoque la conscience ou l'assentiment intérieur

16 «Nous n'avons point les mesures de cette machine immense...», dit le vicaire savoyard. Émile, CEuvres complètes, t. 4, p. 568. 
comme seul juge des conduites et il recommande un "doute respectueux» en matière de doctrine religieuse. Mais il s'empresse d'échapper à cet «état trop violent pour l'esprit humain» qu'est le scepticisme. Puisque $l^{\prime}$ homme "aime mieux se tromper que ne rien croire ${ }^{17}$ ", il faut l'inciter à croire à ce qui correspond à son bien. D'où un certain dogmatisme chez Rousseau, non sur le plan du jugement philosophique mais sur le plan du sentiment. La conscience qu'il invoque n'est pas une recherche singulière, affrontée aux autres consciences, prise dans la diversité des coutumes : c'est la voix de la Nature elle-même. La référence à la conscience ne se donne pas comme une recherche de cette Nature, mais comme sa manifestation indiscutable, car cette conscience est un «instinct divin» garanti par une Providence surnaturelle. D'où la critique très sévère par Rousseau du chaos des coutumes et des opinions sur lequel débouche la pensée sceptique, comme chez Montaigne par exemple ${ }^{18}$.

Cette critique passe avant tout par l'usage particulier qu'il fait des fictions. Dans le cas de celle de l'état de nature, on peut user d'une telle hypothèse en la mettant au service d'une théorie scientifique. L'observation est alors donnée comme fictive, comme une invention commode permettant de pallier les lacunes de l'observation empirique. En fournissant explicitement au lecteur cette projection imaginaire, il est, pour ainsi dire, prévenu qu'il doit chercher en lui, dans un travail nourri par sa propre imagination, des éléments de vérité qui ne sont pas immédiatement accessibles par la science. Science et imagination s'entrefécondent au service d'une observation plus exacte du réel. Toutefois, dans le Discours sur l'inégalité, la fonction de cet entremêlement de la fiction et de la science semble inversée. À côté du choix asséné au lecteur d'écarter au départ tous les faits, Rousseau présente des considérations scientifiques, le plus souvent dans les notes du texte, qui s'efforcent surtout de rendre crédible l'hypothèse de l'état de nature, lequel pourtant, selon Rousseau, n'a peut-être pas existé ${ }^{19}$. Bref, la science semble ici au service de la fiction, comme si c'était celle-ci qui permettait d'appréhender l'essentiel : non pas le réel mais, au contraire, ce qui n'est pas, ce qui devrait être.

La moralisation du réel répond à une exigence constante chez Rousseau qu'exprime cette phrase de l'Émile : les lecteurs «me voient dans le pays des chimères ; moi, je les vois dans le pays des préjugés ${ }^{20} . »$

17 Émile, CEuvres complètes, t. 4, p. 568.

18 Voir Émile, CEuvres complètes, t. 4, p. 598.

19 Discours sur l'inégalité, CEuvres complètes, t. 3, p. 132 et 123.

20 Émile, CEuvres complètes, t. 4, p. 548-549. 
Son effort consiste à arracher ces lecteurs à la prison de ce qui est observable, comme lorsqu'ils préfèrent, contre la leçon du Discours sur l'inégalité par exemple, s'en tenir à l'évidence hobbesienne d'une guerre naturelle entre les hommes. Se méfiant des chimères, ils sont incapables de quitter le pays des préjugés et de s'ouvrir à ce qui constitue la finalité idéale de leur existence, c'est-à-dire ce que Rousseau appelle la nature. Voilà le paradoxe : l'accès à la nature exige le passage par l'artifice d'une fiction.

Cette méthode a été souvent mal comprise au XVIIIe siècle, et depuis ; mais elle n'a pourtant rien d'exceptionnel dans son principe, puisqu'elle ressemble à celle qui inaugure toute la philosophie occidentale chez Platon. Les faits auxquels sont si attachés les lecteurs de Rousseau sont du même ordre que les ombres que les prisonniers de la caverne dans $L a$ République observent sur les murs de leur prison. Ce qu'ils prennent pour le réel n'est en fait que la pâle copie des Idées, qui constituent, si l'on veut, la véritable réalité. Contre les simulacres de ce réel observé par la plupart des hommes, contre la mauvaise fiction que sont les préjugés, Rousseau veut éclairer ses lecteurs par le moyen d'une bonne fiction. Pour les éloigner de leurs habitudes, il faut les tirer au-delà du sensible vers ce qui leur est invisible, mais dont ils gardent en eux les traces et dont ils peuvent se ressouvenir. Les fictions peuvent fournir des modèles idéaux permettant aux hommes de se tenir à distance des évidences sensibles et de s'élever au-delà d'elles vers l'Intelligible. Sur le plan de la vie morale, elles leur permettent de se rendre maîtres de leurs instincts et de régner sur eux-mêmes.

Usant de cette méthode dans sa lecture politique de l'histoire, Rousseau dit de Sparte, par exemple, qu'elle nous apprend «ce que les hommes peuvent être, en nous montrant ce qu'ils ont étée ${ }^{21}$.» En nommant des moments qui échappent à l'histoire décadente et qui permettent de ne pas désespérer du futur, ces références jouent le même rôle que l'état de nature. Mais au lieu d'être des hypothèses que Rousseau s'efforce de rendre crédibles sur le plan des faits, comme dans le Discours, il s'agit de faits présentés comme exceptionnels, élevés au rang de modèles, et qui sont analogues à des fictions par leur portée imaginaire. Le Contrat social s'appuie sur ces modèles du passé et leur donne une traduction pour l'avenir. Pour juger de l'imposture du faux contrat de soumission, décrit

21 «Histoire de Lacédémone», dans Fragments politiques, CEuvres complètes, t. 3, p. 544. «Apprenons s'il se peut à nos contemporains, poursuit Rousseau, qu'un temps a été qu'il existait des hommes et déplorons le malheur et la honte de notre siècle en nous voyant forcés à les chercher si loin de nous.» 
à la fin du Discours par exemple ${ }^{22}$, il ne suffit pas de se tourner vers ce qui a été perdu ; il faut distinguer entre les faits et le droit en projetant ce que serait un contrat légitime. D'où ce qui va paraître encore à beaucoup de lecteurs comme des chimères : celle de la volonté générale ou celle de la démocratie directe ${ }^{23}$.

Par le moyen de cette projection d'un possible, l'homme mal socialisé dans le Discours et l'homme en manque de citoyenneté dans le Contrat peuvent découvrir en eux ce que la Nature leur permet d'espérer. En cessant de consentir aux préjugés, ils peuvent cesser d'être les complices de leur malheur. Mais pour que ce possible devienne opératoire, il faut qu'il pénètre l'imagination des hommes, ce qui ne saurait se faire par le discours rationnel ou par le sermon moralisateur. La capacité de transformer le donné exige des conjectures qui donnent une forme sensible à l'exigence éthique, alors même que celle-ci n'apparaît pas comme réalisable ou raisonnable.

Il s'agit ainsi de faire s'immiscer les modèles de l'état de nature, de la volonté générale ou de l'amour dans la vie intérieure de l'homme moderne ; et il en est de même de l'image de la bonté naturelle de Rousseau, portée par ses textes autobiographiques. Le lecteur est enveloppé par cette vision et entraîné, pour ainsi dire malgré lui, vers un bien qu'il cherche sans le savoir. L'une des conditions de sa métamorphose est qu'il ne s'interroge pas sur ce qui lui arrive, car il doit en quelque sorte se perdre pour se trouver. L'opération exige l'invention d'un nouveau langage qui évite celui de l'interrogation et de l'incertitude. Ce sera le langage du cœur, un langage musical ou, finalement, silencieux, qui permet, non de dialoguer ou de s'interroger avec autrui, mais de sentir avec lui ${ }^{24}$. Bref, l'homme n'est pas arraché à ses préjugés pour être abandonné à l'incertitude: Rousseau façonne son imagination, afin de lui permettre d'habiter un univers idéal qui réponde par avance à ses interrogations.

L'idéal permet de donner du sens, de briser les murs des préjugés, de croire et d'espérer ; le retour critique rappelle que toute croyance a son

23 La manière dont Rousseau se sert de références à l'antiquité, afin d'animer l'imagination de son lecteur par cette idée de démocratie directe, est particulièrement significative. Voir Du contrat social, CEuvres complètes, t. 3, p. 404 et 425-426.

24 Rousseau redoute le silence chez les autres, mais il le présente aussi comme un idéal de communication authentique, telle qu'elle pourrait être pratiquée entre «initiés» dans un «monde enchanté» où l'on échapperait à l'amour-propre. Rousseau juge de Jean-Jacques. Dialogues, CEuvres complètes, t. 1, p. 668-672. 
envers et que les hommes ont tendance à l'oublier en se donnant des certitudes. Tout se passe comme si la conscience démocratique se voulait fidèle à la fois à l'idéalisme et au réalisme, comme si elle s'efforçait d'associer l'élan imaginaire et le désenchantement. Or, la pensée de Rousseau est structurée par cette ambivalence, mais elle ne cesse d'inciter son lecteur à la fuir en se réfugiant dans l'idéal. D'où cet usage dogmatique de la fiction, qui constitue, au bout du compte, le cœur du projet rousseauiste. Un passage du Livre $\mathrm{V}$ de l'Émile l'illustre bien. Rousseau y fait l'éloge du pouvoir des femmes sur les hommes ; il exalte le rôle que joue l'imagination dans l'amour et il déplore que le monde moderne ne soit plus capable de grands élans : «tout n'est qu'illusion dans l'amour, je l'avoue; mais ce qui est réel ce sont les sentiments dont il nous anime pour le vrai beau qu'il nous fait aimer. Ce beau n'est point dans l'objet qu'on aime, il est l'ouvrage de nos erreurs. Eh ! qu'importe? En sacrifie-t-on moins tous ses sentiments bas à ce modèle imaginaire ?» Et Rousseau ajoute : «Nous nous moquons des Paladins! c'est qu'ils connaissaient l'amour et que nous ne connaissons plus que la débauche. [...] il sera toujours grand et beau de régner sur soi, fut-ce pour obéir à des opinions fantastiques ${ }^{25}$ ».

Rousseau explore ainsi minutieusement l'ambivalence de la fiction et du réel ; mais il le fait avec une sorte d'effroi et dans la perspective d'en être délivré, car il ne voit pas dans l'incertitude un art de vivre mais une souffrance que les hommes ne peuvent supporter. Sa tentative radicale de penser, à l'aube de l'ère démocratique, le problème d'une conscience critique d'elle-même, dédoublée et incertaine est finalement conduite dans un esprit qu'on peut appeler pascalien. En ce sens, même si Rousseau (et Pascal) fait partie de ces écrivains dont Tocqueville dit se nourrir chaque jour ${ }^{26}, c^{\prime}$ est bien plutôt l'appréhension de Chateaubriand devant la démocratie qu'annonce la pensée rousseauiste.

\section{PHILIP KNEE}

Université d'Ottawa

26 Tocqueville, Lettre à Kergolay (novembre 1836), dans Correspondance, CEuvres complètes, Paris, Gallimard, 1951, t. 13, vol. I, p. 418. 